\title{
The clinical course of bronchiolitis associated with acute otitis media
} Gila Shazberg, Shoshana Revel-Vilk, David Shoseyov, Anat Ben-Ami, Aharon Klar,
Haggit Hurvitz

\begin{abstract}
Background-Acute otitis media (AOM) is the most common bacterial co-infection of viral bronchiolitis.

Aims-To evaluate the influence of AOM on the clinical course of bronchiolitis.

Subjects-150 children younger than 24 months old, diagnosed with bronchiolitis, hospitalised between December 1997 and May 1999.

Methods-Body temperature, respiratory rate, oxygen saturation, and the need for oxygen supplementation were recorded on admission and daily throughout hospitalisation. Complete blood count, erythrocyte sedimentation rate, and assay for respiratory syncytial virus were performed on admission. All children were examined daily for the appearance of AOM. The clinical course of children with bronchiolitis and AOM was compared to those without AOM.

Results-AOM was diagnosed in 79/150 (53\%) children with bronchiolitis. Most were diagnosed within the first two days of hospitalisation. No significant difference was found in the clinical and laboratory findings on admission and on daily follow up between children with and without AOM.

Conclusions-This 2.5 year prospective study showed no difference in the course of bronchiolitis, whether an ear infection was present or not.

(Arch Dis Child 2000;83:317-319)
\end{abstract}

Keywords: acute otitis media; bronchiolitis; respiratory course

Viral pathogens, especially respiratory syncytial virus (RSV), are a common cause of upper and lower respiratory tract infection in young children. AOM is the most common bacterial co-infection of viral respiratory infection and occurs in $57-67 \%$ of children with RSV infection. ${ }^{1-4}$ In those children with bronchiolitis and AOM, viral antigens, mostly RSV, have been found in middle ear aspirates, either alone or in association with pathogenic bacteria.

Contradictory evidence exists regarding the role of AOM in bronchiolitis. It is not clear whether it is mainly a viral disease, ${ }^{4}$ an integral part of bronchiolitis, or mainly a bacterial disease complicating the viral infection and thus requiring antimicrobial treatment. ${ }^{2}$ Whatever the cause of AOM, its effect on the clinical course of bronchiolitis has not, to the best of our knowledge, been systematically studied. The aim of the present study was to investigate whether AOM, bacterial or viral, influences the clinical course of bronchiolitis.

\section{Methods}

We enrolled children younger than 24 months old, hospitalised with bronchiolitis, in the period between December 1997 and May 1999. Bronchiolitis was defined as an acute viral respiratory syndrome, characterised by cough, tachypnoea (respiratory rate $>40 / \mathrm{min}$ ), dyspnoea, and prolonged expirium with wheezing and/or crackles. ${ }^{5}$ Children with underlying bronchopulomonary dysplasia and congenital heart disease were excluded.

Demographic information obtained on admission included gender, age, birth weight, and gestational age. Clinical data obtained prospectively included body temperature, respiratory rate at rest, oxygen saturation $\left(\mathrm{SaO}_{2}\right)$ in room air, and the need for oxygen supplementation. All these parameters were recorded on admission and daily throughout hospitalisation. Laboratory tests included complete blood count and erythrocyte sedimentation rate on admission. RSV infection was diagnosed by a nasal washing for RSV enzyme linked immunosorbent assay using Abbott TESTPACK RSV (no. 2027-16, Abbott Laboratories, North Chicago, USA). Chest $x$ ray was performed according to clinical indication and reviewed by a radiologist.

All children were examined daily for the appearance of AOM. Criteria for the diagnosis of AOM included the presence of cloudy or yellow discoloration and opacification of the tympanic membrane, combined with at least one of the following indicators of acute inflammation: ear pain, notable redness, and distinct fullness or bulging. ${ }^{6}$ In patients with AOM tympanocentesis was performed. The aspirated fluid was yellow, thick, and purulent; this finding confirmed the diagnosis of AOM. Tympanocentesis was not performed in children older than 1 year of age or when parental consent was not given. Bacterial culture was performed on middle ear aspirate.

The clinical course of children with bronchiolitis and AOM was compared to that of children without AOM. The data were expressed as mean (SD) for each group and the differences between groups were evaluated with the two tailed Student's $t$ test. 
Table 1 Characteristics of children hospitalised with bronchiolitis with and without AOM

\begin{tabular}{llll}
\hline & Total & With AOM & Without AOM \\
\hline Female/male & $66 / 84$ & $40 / 39$ & $26 / 45$ \\
Age $\leqslant 3$ months & 77 & 38 & 39 \\
Age 3-12 months & 63 & 37 & 26 \\
Age $>12$ months & 10 & 4 & 6 \\
Gestational age (weeks) & $39(2.2)$ & $39(2.1)$ & $39(2.4)$ \\
Birth weight (kg) & $2.9(1)$ & $3(0.9)$ & $2.9(1)$ \\
Oxygen supplementation (days) & $2.7(3)$ & $2.8(2.9)$ & $2.6(3)$ \\
WBC $\times 10^{9} / 1$ & $12.8(4.7)$ & $12.9(3.8)$ & $12.7(5.5)$ \\
$\quad$ Neutrophils \% & $46(16)$ & $46(16)$ & $46(17)$ \\
ESR (mm/h) & $46(27)$ & $51(26)$ & $39(27)$ \\
\hline
\end{tabular}

Data expressed as mean (SD). No significant statistical difference between the two groups was detected.

AOM, acute otitis media; WBC, white blood cell; ESR, erythrocyte sedimentation rate.

Table 2 Culture results of middle ear aspirates in children with bronchiolitis and AOM

\begin{tabular}{ll}
\hline Bacteriological findings & No. of patients \\
\hline Haemophilus influenzae & 13 \\
Streptococcus pneumoniae & 12 \\
Haemophilus + S pneumoniae & 1 \\
Staphylococcus aureus & 5 \\
Others & 3 \\
Sterile & 22 \\
Culture not done & 8 \\
Total & 64 \\
\hline
\end{tabular}

\section{Results}

In the period between December 1997 and May 1999, covering three RSV seasons, we enrolled 150 children with bronchiolitis. Their mean age was 20 weeks (median 14 weeks) and male:female ratio 1.7 (84/66). RSV infection was identified in $69 \%$ of the children with bronchiolitis.

AOM was diagnosed in $79(53 \%)$ of the children with bronchiolitis. The male:female ratio was 1 (39/40). Mean age, gender, gestational age, and birth weight were similar in both groups. Children aged 3 month to 1 year were more prone to develop AOM (table 1).

In $69 \%$ of patients with AOM the ear infection was diagnosed within the first two days of hospitalisation; $77 \%$ were RSV positive. Only six children developed AOM after the fifth day of hospitalisation. Two of them were RSV positive.

Tympanocentesis was performed in $73 \%$ (64/79) of children with AOM. Bacterial pathogen was isolated in $36 \%$ of middle ear aspirates. Table 2 summarises bacteriological findings.

The influence of AOM on the course of bronchiolitis was analysed. No significant difference in the clinical and laboratory findings on admission was found between children with or without AOM. Furthermore, the daily follow up of body temperature, respiratory rate, $\mathrm{SaO}_{2}$ in room air, and oxygen supplementation showed no difference between the two groups (table 3).

Seventy one children with bronchiolitis did not have AOM. Thirty three $(46 \%)$ of them received antibiotic therapy for pneumonia, leucocytosis, or suspected neonatal sepsis. Excluding these children and comparing the remaining 38 children without AOM who did not receive antibiotics with the group of children with AOM revealed similar respiratory course in terms of $\mathrm{SaO}_{2}$, respiratory rate, and need for oxygen supplementation.
Table 3 Five days follow up of body temperature, arterial haemoglobin $\mathrm{O}_{2}$ saturation $\left(\mathrm{SaO}_{2}\right)$, and respiratory rate

\begin{tabular}{lllll}
\hline Day & $\begin{array}{l}\text { AOM } \\
(+-)\end{array}$ & $\begin{array}{l}\text { Temperature } \\
(\mathrm{C})\end{array}$ & $\begin{array}{l}\text { Respiratory } \\
\text { rate }\end{array}$ & $\mathrm{SaO}_{2}$ \\
\hline 1 & + & $38.02(1.0)$ & $56.4(11.7)$ & $90.2(5)$ \\
& - & $38.05(0.9)$ & $59.08(14.7)$ & $90.7(4.11)$ \\
2 & + & $37.56(0.8)$ & $50.2(9.6)$ & $91.95(2.7)$ \\
& - & $37.38(0.75)$ & $53.08(13)$ & $91.1(4.28)$ \\
3 & + & $37.32(0.69)$ & $49.8(10.8)$ & $92.3(3.8)$ \\
& - & $37.28(0.8)$ & $51.8(14.5)$ & $91.1(6.24)$ \\
4 & + & $37.1(0.56)$ & $50.3(9.6)$ & $92.4(2.7)$ \\
& - & $37.2(0.7)$ & $48.6(10.7)$ & $91.7(3.4)$ \\
5 & + & $37.07(0.5)$ & $48.4(8.58)$ & $92.8(2.9)$ \\
& - & $37.1(0.63)$ & $46.8(11.8)$ & $92.5(2.7)$ \\
\hline
\end{tabular}

Data expressed as mean (SD). No significant statistical difference between the two groups was detected.

$\mathrm{AOM}$, acute otitis media; $\mathrm{SaO}_{2}$, oxygen saturation.

\section{Discussion}

AOM is found frequently as co-infection in infants and young children with bronchiolitis. In the present study, $53 \%$ of children with bronchiolitis had AOM, most of them aged 3 months to 1 year..$^{7-9}$ Tympanocentesis which was performed in the majority of infants confirmed the diagnosis of AOM. Thirty six per cent of middle ear aspirates revealed bacterial pathogens. Culture negative aspirates could have resulted from previous antibiotic treatment, microbiological failure or a viral ear infection. ${ }^{10}{ }^{11}$

To the best of our knowledge, the effect of AOM on the clinical course of RSV bronchiolitis has not been evaluated objectively. We anticipated that the ear infection would exaggerate the clinical course of the bronchiolitis. Nevertheless, our present 2.5 year prospective study, including daily follow up of body temperature and respiratory parameters, showed no difference in the course of the disease whether an ear infection was present or not.

It is worth noting that no clinical or laboratory parameters could predict AOM on admission or later in the course of the bronchiolitis. The ear infection usually appeared during the first two days of hospitalisation. ${ }^{7}$ It might be that the early diagnosis and treatment with both antibiotics and tympanocentesis modified the potential deleterious effect of this infection on the clinical course of bronchiolitis.

Further studies are needed in order to evaluate the influence of AOM on the clinical course of bronchiolitis and to determine the therapeutic recommendations for this group of young infants.

1 Ruuskanen O, Arola M, Putto-Laurila A. Acute otitis media and respiratory virus infections. Pediatr Infect Dis $\mathcal{F}$ 1989;8:94-9.

2 Andrade MA, Hoberman A, Glustein J, Paradise JL, Wald ER. Acute otitis media in children with bronchiolitis. Pediatrics 1998;101:617-19.

3 Henderson FW, Colier AM, Sanyal MA. A longitudinal study of respiratory viruses and bacteria in the etiology of acute otitis media with effusion. $N$ Engl 7 Med 1982:306: 1377-84.

4 Ruuskanen O, Arola M, Heikkinen T, Ziegler T. Viruses in acute otitis media: increasing evidence for clinical signifiacute otitis media: increasing evidence for

5 Gruber WB. Bronchiolitis. In: Long SS, Pickering LK, Gruber WB. Bronchiolitis. In: Long SS, Pickering LK,
Prober CG, eds. Pediatric infectious diseases, 1st edition. Prober CG, eds. Pediatric infectious diseases,
New York: Churchill Livingston, 1997:246-50. 
6 Weiss JC, Yates GR, Quinn LD. Acute otitis media: making an accurate diagnosis. Am Fam Physician 1996;53: 1200-6.

7 Koivunen P, Kontiokari T, Niemela M, Pokka T, Uhari M. Time to development of acute otitis media during an upper respiratory tract infection in children. Pediatr Infect Dis $\mathcal{F}$ 1999;18:303-5.

8 Heikkinen T. Ruuskanen O. Temporal development of acute otitis media during upper respiratory tract infection. Pediatr Infect Dis f 1994;13:659-61.
9 Arola M, Ruuskanen O, Zeigler T, et al. Clinical role of respiratory virus infection in acute otitis media. Pediatrics piratory virus infect

10 Sarkkinen H, Ruuskanen O, Meurman O, Puhakka H, Virolainen E, Eskola J. Identification of respiratory virus antigens in middle ear fluids of children with acute otitis media. F Infect Dis 1985;151:444-8.

11 Chonmaitree T, Howie VM, Truant AL. Presence of respiratory viruses in middle ear fluids and nasal wash specimens from children with acute otitis media. Pediatrics 1986;77:698-702.

\section{Coke gets in your bones?}

Why would drinking Coca Cola and other fizzy drinks make children more prone to bone fractures? That seems a difficult question to answer but a study in Boston, Massachusetts (Grace Wyshak. Archives of Pediatrics and Adolescent Medicine 2000;154:610-13), has provided empirical evidence linking the intake of these drinks with a history of fractures in teenage girls.

A total of 460 teenage schoolgirls took part in a retrospective questionnaire survey in which they were asked, among other things, about their physical activities, whether or not they drank carbonated beverages, and whether they had ever had a bone fracture. They were not asked about the quantities of the various drinks they consumed. About $80 \%$ replied that they habitually drank carbonated drinks, mostly colas. Twenty per cent reported having had a fracture, some in early childhood.

In the whole study group, drinking carbonated beverages increased the odds of having had a bone fracture threefold. In physically active girls, but not inactive girls, drinking colas increased the odds of fracture fivefold compared with drinking non-carbonated drinks or non-cola carbonated drinks. Drinking both cola and non-cola carbonated drinks increased the odds sevenfold compared with drinking only noncarbonated drinks.

It all seems rather strange. No explanations are offered other than observations that cola drinks have a high content of phosphoric acid and American teenagers have been drinking about $40 \%$ less milk than they used to. The cross sectional design of the study can't prove cause and effect and there are no data about bone mineral density. It's an important subject though, and developments will no doubt be awaited with interest.

ARCHIVIST 